\title{
Curiosidad, disciplina, divulgación. Investigar en arquitectura
}

\author{
Joaquín Ibáñez Montoya \\ Universidad Politécnica de Madrid, España
}

Recibido: 3 de setiembre de 2013 / Aprobado: 26 de noviembre de 2013

Investigar desde la arquitectura implica asumir una postura disciplinar más amplia pero también de respuesta acelerada, que necesita adecuar sus instrumentos para pensar. El estudio y la docencia sobre el hecho arquitectónico fueron siempre un ámbito en formación no finalista, que redibuja ahora un protocolo innovador unido a su enseñanza. El objetivo de la investigación es siempre producir utilidad social y aporte de conocimiento. Los planes docentes, que se encuentran cada vez más asociados a la investigación, estimulan la estrategia de un método que necesita dialogar. La pedagogía debe encontrar las razones que hacen del conocimiento una arquitectura verdadera que, quizás, precisa desplazar su centro.

estratigrafía, evaluación, innovación, inversión, desplazamiento

\section{Curiosity, discipline, disclosure. Research in architecture}

From the present perspective, architecture research implies assuming a wider disciplinary position, but also an accelerated response that needs to adapt its instruments to thought. The study and teaching of the architectural fact was always a non-finalist training area that redesigns now an innovative protocol together with its teaching. The objective of research is always to produce social utility and bring knowledge. The teaching plans, which are more and more associated with research, stimulate the strategy of a method that needs dialogue. Through its impulse, pedagogy must find the reasons that make of knowledge a real architecture that may need to displace its center.

stratigraphy, evaluation, innovation, inversion, displacement 


\section{CURIOSIDAD}

En un marco de reflexión contemporánea sobre la investigación en arquitectura parece muy adecuado traer a un primer plano, para comenzar, aquella pregunta formulada por el año 1939 por el compositor Igor Stravinsky: ¿qué utilidad podía suponer el debatir continuamente sobre el principio mismo de la operación de la crítica? En su caso, musical ¿para qué fatigar al autor, meditaba, sobre "por qué" había elegido tal o cual asunto, tal argumento, tal forma? ¿A dónde conduce hostigarle de manera constante en vez de buscar mejor, uno mismo, el "cómo" y establecer así las razones del fracaso o del éxito de la indagación? La poética, nos recordaba, sugería una estrategia de ideas y de trabajo personal. La propuesta del Juan de Mairena machadiano, en su diálogo del hombre, del hombre con su tiempo en tanto que pescador de "pescados vivos" (Machado, 1971). Un hacer actualizado. Lo apuntaba el también poeta John Oldham:"tome un tema conocido e invéntelo bien"; haga una recreación efectiva sobre las presentes aproximaciones transarquitectónicas. Y, sin embargo, realizar la operación de indagación que motiva este texto no es tarea fácil. Simplemente, el iniciarla, demanda un sinfin de aclaraciones previas para evitar errores o malentendidos.

No es esta investigación, pues, solo un ejercicio de espacio sino de tiempo; se trata de una tarea de "estratigrafias" sobre lo construido. Desde la mirada del presente, investigar en arquitectura implica asumir una postura amplia pero también acelerada que necesita, lógicamente, adecuar todos sus instrumentos de pensar. Su argumento crucial, estratégico, sobre el tradicional conocimiento precisará, además, de una "evaluación" controlada desde la que sumar las diversas escalas que exige su contexto presente. Investigar aquí supone, en tercer lugar, incorporar condiciones artísticas a las científicas habituales; incorporar las razones creativas del nuevo milenio para enlazar su ambivalencia entre ciencia y creación e incorporarla a las condiciones científicas y políticas propias. Añadirlas en definitiva a unos programas actuales de doctorado de rango cosmopolita, de gestión de redes y grupos, con factores objetivos de acreditación del profesorado y aumento de las relaciones con el tejido empresarial y la realidad social al que se ha añadido, en las últimas décadas, una cultura digital emergente que supone algo más que un simple cambio de sistemas en la apropiación de conocimiento y de su construcción. 
La investigación, de ese modo, define un lugar propio al manejarse desde la arquitectura. Un lugar en el que se construye una nueva tradición que identifica su proceso de proyectación como un vínculo entre aquel y su relato, entre la imagen y el deseo; un pasado recuperado como desencadenante. En este paisaje extensivo, el campo de la investigación disciplinar pretende traducir sus vías habituales de mente, lugar y sociedad en innovadores soportes de entrecruzamiento entre virtualidad y realidad. Entre esta actual espacialidad, las condiciones temporales de su enunciado construyen un innovador re-significado cultural que permite revisar las bases sobre las que habita la sociedad. Una relación con un paisaje antropizado que expone un repertorio de preguntas pertinentes; un recinto de transformación del hecho arquitectónico. Es cierto que siempre fue un ámbito en formación, no finalista, y ello facilita su papel para permitir a sus estudiosos orientarse en él de una manera abierta, según muy diversas capacidades y caminos. En esta profundización presente, caracterizada por la condición autorreflexiva aquí expuesta, se desarrolla su indagación. Enuncia una "disciplina de acción”, un habla y estructura, que redibujan un protocolo moderno intrínsecamente unido a su enseñanza.Volveremos sobre ello.

Lejos de limitar, su indagación abre un interfaz entre cuerpo y mente. Como un recinto de exploración de relaciones selectivas nos propone mecanismos de abordaje, en un panorama ilimitado, de posiciones de lectura e interpretación. Desde una mirada innovadora, oblicua, transversal, de sorprendentes estímulos, avanza un proceso de "luces" ante la dificultad de sus retos. De manera colectiva, la condición de la investigación en arquitectura establece los momentos sobre los que se deben analizar las condiciones de sus fronteras presentes; un patrimonio común que ayudará a croquizar las trayectorias desde las cuales orientar sus pliegues (Deleuze \& Guattari, 1994). Una especie de cajón de señales cibernéticas. Pero, a la vez, colaborará también a detectar su capítulo de carencias. Un ejercicio de interrogantes se deriva de su desarrollo. En este presente postindustrial, de tiempos mutantes, exige no solo adecuar materias sino también políticas para poderlas ejecutar.

Asegurar la satisfacción de su curiosidad congénita requiere un trabajo continuo, crítico. Requiere definir los medios con los cuales evaluar qué y cuánto da de sí su proyecto de investigación, e intentar desvelar sus 
evoluciones, sus futuros comportamientos, a través de las proyecciones de sus lógicas singulares para adelantar parámetros con los cuales enriquecer el impacto de las nuevas tecnologías sobre la disciplina. Sin duda, sus propias formas de representación, contemporáneas, influyen en su radicalidad actual y por tanto en sus modos de ser pensada. La arquitectura, en sus métodos, más que en su propia concepción, no puede ni es ajena a todo ello; sus conclusiones sobre modelos y productos o sobre la misma organización del trabajo, sobre su producción asociada, interdisciplinar, acota los aspectos más atractivos de su potencial investigador.

Todo va a depender, lógicamente, de la capacidad de integración de los grupos de investigación que las universidades aporten al trabajo. En España, en arquitectura, los grupos reconocidos lo son solo desde hace pocos lustros; todavía no tienen una relación muy directa y fluida con la investigación general. En estos últimos años en Madrid, en la Politécnica, los primeros programas de maestrías oficiales para doctorado, con buen éxito de demanda y de resultados, han permitido que sus alumnos se inserten en ellos; ahora vamos a comenzar con las maestrías de Centro para Iberoamérica y para China. En español, para formar doctorandos, y en inglés, con un carácter más profesionalista.

Es de esperar que el nuevo ciclo de posgrado "Bolonia" también se convierta en otro pilar de una formación renovada del arquitecto. Que suponga un espacio para la excelencia y para la formación de un profesorado con la escala universitaria adecuada. Que sea un lugar donde se puedan generar áreas de interés específicas para enfrentar sus problemas estructurales e identificar sus estrategias en un mundo progresivamente inabarcable y cambiante. Aportar a la sociedad postindustrial, en suma, un plus de conocimiento propio de la universidad, no solo reproducción de saber. Un espacio para movilizar los saberes "innovadores" en arquitectura" ${ }^{1}$

El objetivo de cualquier investigación es, siempre, producir utilidad social y aporte de conocimiento. Es preciso, por tanto, asociar la arquitectura a las nuevas

1 Es de interés conocer la puesta en marcha de redes académicas por la Universidad Politécnica de Madrid, como el Proyecto PHI Patrimonio Histórico + cultural Iberoamericano, del cual el autor de este artículo es investigador responsable, junto con los profesores José Manuel Páez, Fernando Vela y Ernestina Manesalvas. 
demandas; sin olvidar las materias clásicas. Y para ello es necesario incluir a los profesionales de su docencia en la investigación, corrigiendo su falta de experiencia en este campo. Existen aquí quienes proponen incorporar voces externas a la disciplina para hacerlo más rápido; metodólogos que enseñen a evaluar y a aplicar criterios de calidad a sus egresados; quizá también del mundo empresarial. Estoy hablando desde la universidad pública; no creo en otra. Y por tanto, bienvenidos sean. En Holanda hace tiempo que los doctorados en arquitectura dependen de los programas de investigación, lo que, a mi entender, es muy lógico; supone un futuro para asegurar una enseñanza superior en sistemática evaluación, particularizada y ajustada a las aceleradas transformaciones. Los programas de estudio cubren así registros temáticos cada vez más amplios y variados, que van a exigir todo nuestro tiempo y dedicación; pero más que nada toda la imaginación disponible ante estas culturas con las que se encuentra la investigación del arquitecto.

Por otra parte, en todas las escuelas o facultades de arquitectura, al menos las europeas, podríamos decir que se está de acuerdo en su centralidad sobre el proyecto; parece lógico. Aunque no existe una tradición investigadora suficiente sobre él, supone un área común; la más genuina. Aun con las perversiones que sobre ella hicieron las vanguardias del siglo pasado y muchos de los llamados pioneros. Parece imprescindible, pues, afirmar sus límites para evitar confusiones fáciles. Porque el proyecto profesional es otra cosa; no fue nunca un ejercicio de tesis de investigación como algunos cortoplacistas pretenden: sería un error. Otra cosa es que pueda ser un soporte válido, por su calidad, o que sobre él se pueda levantar un protocolo adecuado para su doctorado; ser un desencadenante.Todo proyecto arquitectónico tiene siempre algo de investigación, pero, lógicamente, su envergadura de cara a una investigación o una tesis no tiene el nivel adecuado; no es su fin. Cuestión de objetivos: de qué se pretende.

\section{DISCIPLINA}

La disciplina es la base del protocolo de la investigación académica. Desarrollar sus pasos implica no solo hacer balance sino evidenciar lo atractivo de esta reactivación iniciada en arquitectura. En su siempre relativamente mundo autónomo, "hacer del vicio, virtud" recurriendo a una cierta mecánica 
de feedback muy propia de su sistemática habitual supone ahora sumarse a un debate intrínsecamente vinculado a su práctica; establecer una discusión enmarcada por la dudas y por las posiciones encontradas ${ }^{2}$. No es tema fácil, ya ha sido dicho. Nadie lo espera tampoco. El ejercicio de su definición "a futuro" va a necesitar por supuesto de muchos foros y de diversas escalas. De muchas reflexiones como esta sobre sus experiencias primigenias. Del mismo modo, tampoco es sencilla su inserción en el turbulento ambiente de los recintos de la docencia. Lo que desde luego no es un problema administrativo.

Enfrentar su reto es crucial, imprescindible ahora. El perfil de la investigación en la pedagogía de la arquitectura, en estos últimos años, se anuncia como una oportunidad de renovación académica esencial. Es dificil pensar en hablar de calidad, innovación, sin un convincente ciclo de posgrado; su perfil ha pasado a transformarse, con rapidez, en un periodo de excelencia universitaria. Como condición científica, la arquitectura puede mostrar, demostrar, demostrarse quizá, que investigar no significa solo un trabajo que se va a realizar en laboratorios dotados de costosísimos aparatos que, en algunos casos - inclusolo pudieran ser; parece obvio que su perfil responde a condiciones más específicas. Como disciplina, su indagación atiende abanicos muy extensos, que han comprometido siempre a su propia cultura; así ha sido entendida en los dos últimos siglos. Significa una política de selección de materias donde primará su valor comunicativo y que, como se ha señalado, marca una nueva inteligencia ante la histórica curiosidad que estimula al ciudadano.

En todo caso, como proceso de investigación, la arquitectura, en sus resultados o conclusiones, está claro que no debe entrar como una forma estética; ni tampoco lo hará como su producción ejecutiva. Como muy bien explica el profesor Prada Poole (1998) —utilizando el ejemplo del manuscrito de trabajo de Alberto Durero, de la Biblioteca de Dresde - la obra de arquitectura no es una obra de arte. Lo sería, quizá, su grabado final; por el contrario, Prada entiende de hecho como investigación, o un principio de ella, el cuaderno de tal nombre. Una tesis doctoral en este campo puede contener o apoyarse en un proyecto siempre que lo haga con una lectura generalista, no situacional,

2 Consúltense, por su interés, los diversos Congresos Europeos sobre investigación Arquitectónica y Urbanística (EURAU) especializados en esta temática en el ámbito europeo. 
y en clave de aportación metodológica, como una verificación de hipótesis. La finalidad de su trabajo, no hay que olvidarlo, es demostrar puntualmente a la comunidad científica, garantizarla, que se ha alcanzado un cierto grado de madurez intelectual sobre la profesional pura; que se dispone de unos ciertos hábitos que aseguran su capacidad como investigador; que se está en disposición de aumentar el conocimiento propio y el de los demás, y de poder hacerlo de manera transmisible y verificable.

El asunto es claro. Establecer este discurso científico orientado por la anticipación que tanto amaba otro compositor musical, John Cage, en esta "crisis de la investigación", tiene, para la arquitectura en concreto, que ver con muchas cosas; primero con la disciplina misma. El reconocimiento de su profesional no es el mismo que el del investigador.Adquisición de conocimiento, y no solo ciencia, en este sector no es solo acumulación de saberes, aunque fueran de acepción diversa. Porque, en definitiva, subsisten preguntas por responder que aquí importan, como ¿cuál es la diferencia entre la formación profesional y la investigación?, ¿cuáles son los objetivos específicos característicos de esta última?, ¿por qué es necesaria una investigación arquitectónica para nutrir la práctica profesional? y ¿cómo? Parece esencial definir los términos de su autonomía presente como investigación para además referenciar su propia práctica profesional.

Supone movilizar saberes espaciales o preguntas sin concluir y que se pueda ir "cuanto lejos se quiera", con la condición de tener claro que el objetivo de la formación profesional no es otro que producir un conocimiento de universalidad, objetividad, verificación; de transversalidad e hibridación (algo que los textos del propio Vitrubio ya avanzaban). Como indagación científica, propone una posible ruptura epistemológica. Desde la distancia crítica de la mirada del arquitecto respecto del historiador, por ejemplo, parece claro que, con todos sus riesgos, es preciso establecer un auténtico "núcleo duro" para reconstruir, en su entorno, su proyecto. Implica comprometerla con un proceso de generación de áreas de interés para caracterizar su programa de estudios. De este modo, los planes docentes se encuentran cada vez más asociados a la investigación, como a la cooperación, por cierto; de ello se pueden derivar consecuencias de enorme interés en la formulación de esta reflexión.

Los centros universitarios intentan ser, lógicamente, cada vez no solo más disciplinados al respecto sino más independientes. En un panorama presente muy 
competitivo estimular estos objetivos incitando el enlace entre investigadores y docentes de una manera transparente y divulgativa parece algo perfectamente acorde con el estatus de una politécnica. Debería permitir enfrentar este mundo de colaboraciones y acuerdos por explorar. Responder a las demandas citadas de la realidad contemporánea, en parte desconocidas. Hacerlo desde epígrafes en que los saberes clásicos del arquitecto encajan hoy de una manera básica, insuficiente. El protocolo de la investigación, que siempre interroga, al fin y al cabo, sobre hipótesis por contrastar, facilita obtener resultados. Tras una definición de objetivos claros y concretos, estrechamente relacionados con conclusiones congruentes, escuetas y jerarquizadas, el acto de pensar, como algo inseparable de la modernidad, implica "ser en el tiempo" en términos hegelianos; y tendrá éxito.

El valor de toda esta innovadora "geografia de investigación" ahora iniciada estribará, con su rigor y coherencia interna, en riqueza de método. Es el extremo opuesto a la hipótesis subjetiva, discutible. El tiempo de su desarrollo persigue obtener un repertorio de conclusiones transmisibles, reproducibles, sin barreras lingüísticas, que faciliten conocer qué se hace en el Perú o en España, y viceversa. Es preciso no solo evitar el acto fallido, dramático, que nos recordaba Steiner en su tesis, sino establecer las redes de documentación, auténticas plataformas iberoamericanas, de un verdadero cuerpo común científico para el uso de las estrategias actuales.

Además, al diferenciar los dos grandes capítulos que permiten iniciarlo, definición de problemas y método, el primero, en su introducción, lo hará justificando un "estado del arte" al día de hoy. Hablamos de un diálogo entre razón intelectual y sentimiento. Una de las ventajas ciertamente de campos autónomos como la poesía o la ciencia, de los que se nutre por igual la arquitectura, es que pueden acentuar las condiciones sociales que los justifican, desde múltiples recursos. Es mérito de Thomas Kuhn hacer de su "teoría de rupturas y revoluciones" un criterio de trabajo.

Como ciencia, la disciplina aquí tratada es ontológicamente indiferente: "la ciencia no piensa, y además es colectiva”. Defiende en sus textos el valor del método científico de la investigación que pasa por la disciplina, pues todo proyecto debe ser capaz de predecir una serie de hechos desconocidos (Lakatos, 2002). Toda propuesta sin corroboración carecería de capacidad explicativa; y no 
sería científica (Popper, 1973). Precisa de su verificación pautada. Ser profesional en la investigación depende de demasiados agentes para descuidar su orden.

El paisaje cultural que hoy piensa el arquitecto que investiga en una "sociedad por venir sin fronteras", que todos intuimos, de grandes infraestructuras, de problemas críticos medioambientales y habitacionales, exige visualizar una nueva disciplina. Por encima de todo, necesita dialogar. La investigación de su geografía metafórica va a posibilitar el compromiso de toda una generación en el diseño de un mapa desde el cual orientarnos; y esperar que produzca los efectos aceleradores que su condición estimulante conlleva. Porque la clave de su interés contemporáneo es permitir seguir interrogando. Hacerlo sobre un repertorio de hipótesis, estratégicas, por contrastar, para obtener resultados desconocidos y éticos desde su adecuación a un tiempo marcado por abundancia y exceso informativo. Un panorama que recomienda prudencia ante su obligada incertidumbre, que se asemeja al de los biólogos en su examen atento de la materia y en su continua comparación de cada muestra con las demás.

\section{DIVULGACIÓN}

El mundo de la investigación arquitectónica finaliza con un potente espacio para la divulgación de resultados. Un punto donde su excelencia es clave, en lo aportado y en lo soportado. Abocado a enfrentar dificultades singulares como disciplina, pues esta no fue considerada institución realmente universitaria sino hasta mediado el siglo pasado. Como disciplina se inserta mal en las clasificaciones tradicionales. Su protocolo, a la hora de la investigación, es todavía incierto. En muchos casos, es entendida como un "lugar de formación en la investigación" más que "un lugar — exactamente- de investigación”. No están ni suficientemente claros sus criterios de evaluación, ante los clásicos, ni los repertorios indexados de las publicaciones periódicas para compartir sus resultados, ni existe tal cultura. Es bien cierto que se está realizando un esfuerzo encomiable y acelerado en esos últimos años en virtud a su necesidad. El mundo de sus publicaciones, en general, ha estado mucho más asociado con la información descriptiva profesional y hasta con la de una prensa del "corazón” académica.

La condición práctica de su grado pesa quizá demasiado todavía. Es un panorama que se abre poco a poco a un sistema de evaluaciones múltiples en 
su apropiación del conocimiento y de sus métodos. Es posible que los cambios del Plan Bolonia, por ejemplo en España, en estos momentos en progresiva implantación, alteren muchas de estas culturas. Además, los criterios en la indagación y su pedagogía, en arquitectura, son muy diferentes; se mezclan ciencias introspectivas con ciencias de la representación. La condición experimental, parte importante de su razón de ser disciplinar, exige atender a estas visiones diferenciadas. Los arquitectos en uno $\mathrm{u}$ otro plano son algo más que técnicos científicos. Aunque es cierto que la creación es una condición que puede ser leída también como aportación de su conocimiento específico, que distingue a la formación del arquitecto como aproximación plural, multidisciplinar. En realidad todo esto exige un esfuerzo por parte de los centros en el sentido de buscar su inserción en tal interdisciplinariedad.

La investigación en la enseñanza del arquitecto quizás necesita de equipos no solo integrados por sus profesionales mismos. Su indagación precisa de una rectificación endogámica. De una movilidad asociada con la sociedad que la envuelve y, a la par, vaya acompañada del estudio filosófico, heurístico, para no ir a ciegas. En la medida en que anticipa hechos teóricamente nuevos esta atractiva labor de fijar límites, de ajustarlos en el tiempo y espacio, nos acerca a la dificultad de un compromiso. Dividida entre episteme, conocimiento probado, y doxa, o simples opiniones, su conocimiento con la primera acepción toma el camino correcto; supone aumentar aquella primera concepción y disminuir la segunda. Evaluación y heurística construyen aquí un protocolo característico entre dos temporalidades; definen una táctica propia del proceder del arquitecto que evoca e imagina, que propone una secuencia, un abanico de hipótesis, integradora de resultados y de anticipaciones (Ibáñez, 2008). La evaluación de su metodología al realizar esta verificación de su proyectación, monitorización, nos trae a la memoria la tesis de la "La arqueología del saber" de Michel Foucault. Concluye una mirada cuya base es el "proyectar" y el "proyecto", el "saber hacer" y el "hacer kantianos", que estimula estos principios de valoración y heurística. Una medida de permanente análisis, de reflexión, que pretende apoyar con su experiencia y diálogo una revisión desde la investigación a su vez verdaderamente útil para su pedagogía.

Porque el objeto de todo doctorando o investigador es, en general, naturalmente, 
la enseñanza; y por tanto este es uno de sus retos. Su proyecto, además de bello, debe ser pedagógico, propedéutico; debe tratar de sus hipótesis, —ahora en crisis casi de supervivencia-, como una oportunidad. ¿Cuánta arquitectura realmente de nueva planta se precisa? Una "oportunidad de oro" para revertir respuestas al tejido colectivo social en este fin de ciclo largo de una modernidad revisada en el 68, hace ya cuarenta y cinco años. No hacerlo, o hacerlo de manera inadecuada o tímida, podría suponer perder el "tren histórico" con el que ha convivido desde el comienzo del Renacimiento.

El debate ofrece pues, para concluir, una opinión que, de nuevo de la mano de Foucault, sugiere resultados comprometidos con la revisión disciplinar. Describe una estrategia de espacios por descubrir recordando las recomendaciones del Colegio de Francia de hace unos años. En su reclamación incluía ya, entonces, no solo un mayor apoyo a los grupos de investigación sino a la pluri-anualidad de sus proyectos; pero también a cuestiones muy importantes e inteligentes como su inter-generacionalidad y transparencia y su apertura cosmopolita -internacional y europea- en el tiempo biopolítico que inauguraba con el fin del siglo pasado. Se esforzaba por avanzar el potencial de un proyecto contemporáneo para romper con todo orden cronológico de la historia.

Y naturalmente con el esfuerzo por dotarse de instrumentos como la investigación en arquitectura y urbanismo. Esencialmente lo veía como una "constitución de conocimiento". Frente a la tradicional actitud deductiva del proyecto de arquitectura, objeto central de esta reflexión, plantea "invertir" su estrategia de una manera diferente. Enunciar las cuestiones de nuestro tiempo, su repertorio de codificaciones para aportar medios en los que asentar sus conclusiones. Diseñar sus escalas, enmarcar la labor ahora emprendida desde un escenario colectivo diferente que arranca desde atrás un salto cualitativo, más que acumulativo, verdaderamente audaz, para averiguar el sentido de las convergencias crecientes en mundos muy distantes y así, paulatinamente, incorporarlas más y más como leales aliados. Asuntos estos que tienen que ver con los problemas de revisión del protocolo que Antonio Miranda racionaliza. En un sentido spinoziano, más que de interpretación, este reclama ejercer la acción como una negentropia, como una acción militante opuesta al devenir del tiempo.

Como curiosidad no del todo desvelada, disciplina y sobre todo divulgación, 
la pedagogía que se deriva de la nueva estrategia de la indagación académica debe encontrar sus razones para hacer en una verdadera arquitectura. En contextualizar las múltiples preguntas relativas a su "cuestión doctoral" y a la naturaleza de las producciones de su investigación en una cultura urbana global renovada en sus objetivos, reexaminando sus territorios, sus métodos y, de manera general, su naturaleza y pertinencia (Glaeser, 2011). Y como en otras materias, quizás asumir el "desplazamiento de su centro" hacia alrededores inmediatos en la búsqueda de nuevas respuestas.

Como pedagogía, como actividad pautada, se interesa por enunciar un itinerario cognoscitivo más actualizado como objetivo para aportar medios en los que asentar tanto su reflexión como, sobre todo, su futuro. Supone una labor colectiva que "arranca donde alguien la dejó" para, de nuevo, concluir de manera abierta en sus resultados. Abordar hoy la investigación en arquitectura, su propia tesis doctoral, con garantías de estructura y de tiempo no deja de ser un trámite vital. Requiere, en realidad, perfilar la formación de un nuevo tipo de profesional. ¿Quizá puede estar la arquitectura, con la investigación, abriéndose así a nuevos campos de competencia?

Volviendo a Igor Stravinsky, con quien empezamos. Es correcto recordar, una vez más, como su ensayo docente y la cuestión de trabar conocimiento bajo el signo austero del orden y la disciplina siempre fue, para él, parte del problema. No hay que olvidar que el autor buscaba un camino cierto en este reino de la disimilitud contemporánea que inevitablemente estaba, y está, conducido por razones de "estructura". La arquitectura, como indicaba aquel sabio chino, para la música, unifica; como estructura, su pensamiento verifica tiempos de ambigüedad y aceleración. No hacerlo, o demorarlo, supondría perder, a mediano plazo, el perfil querido de su autonomía.

\section{REFERENCIAS}

Deleuze, G. y Guattari, F. (1994). Mil mesetas.Valencia: Pretextos.

Glaeser, E. (2011). El triunfo de las ciudades. Madrid:Taurus.

Ibáñez, J. (2008). Los archivos: cómo construirlos. Gijón: Ediciones Trea. 
Kuhn, T. (2006). La estructura de las revoluciones científicas. México: Fondo de Cultura Económica.

Lakatos, I. (2002). Escritos filosóficos. Madrid: Alianza Editorial.

Machado, A. (1971). Juan de Mairena. Madrid: Castilla.

Miranda, A. (1999). Ni robot ni bufón.Valencia: Pretextos.

Popper, K. (1973). La miseria del historicismo. Madrid: Alianza Editorial.

Prado Poole, J. M. (1998). La investigación en el proyecto. Madrid: Mairea.

Steiner, G. (2003). Lecciones de los maestros. Madrid: Siruela.

Stravinsky, I. (1939). Poética musical. Seis lecciones en la Universidad de Harvard. Cambridge: Hardvard College. 